\title{
Metabolic Syndrome (MetS)
}

\section{Mra Aye*}

Department of Medicine, UniKL Royal College of Medicine, Perak, Malaysia

*Corresponding author: Mra Aye, Clinical Professor/Internist, Department of Medicine, Melaka Manipal Medical College, Jalan Hampur, Bukit Baru 75150, Malaysia; Email: mraaye@hotmail.com

Received date: September 15, 2014; Accepted date: September 16, 2014; Published date: September 23, 2014

Copyright: (c) 2014 Aye M. This is an open-access article distributed under the terms of the Creative Commons Attribution License, which permits unrestricted use, distribution, and reproduction in any medium, provided the original author and source are credited.

\section{Editorial}

The clinical cluster of hypertension, cardiovascular disease, hyperlipemia, hyperuricemia and type 2 Diabetes had been recognized by physicians for many decades, described as syndrome $\mathrm{X}$ and finally metabolic syndrome (MetS). A few decades ago, investigators noted this syndrome in the alarming pandemic of obesity and type 2 diabetes. Different definitions of metabolic syndrome had been developed by the World Health Organization (WHO), the National Cholesterol Education Program me Adult Treatment Panel 111 (NCEP-ATP111) and by the International Diabetes Federation (IDF). High Triglycerides, low HDL-C, hypertension, raised fasting plasma glucose, and central obesity are essential factors. Obligatory factors such as obesity $(\mathrm{BMI} \geq 30)$ and high waist circumference with ethnic specificity were required by WHO and IDF criteria respectively. Cutoff points of systolic and diastolic blood pressures, HDL-C and fasting plasma glucose of WHO criteria were required to be adjusted; and cutoff points of waist circumference for males and females of IDF were required to be standardized with European and non-European populations, South Asians and South-East Asians of WHO and IDF criteria. Later, three out of five criteria was introduced by NCEP ATP111 instead of obligatory requirements plus two out of four criteria used by WHO and IDF. To be practical for application to public health sectors, MetS has been defined by at least two criteria such as hypertriglyceridemia-waist and hypertensive-waist by validation to IDF criteria definition. Some elements of MetS are noted to be clustered more frequently in some ethnic populations. Individuals without type $2 \mathrm{DM}$, obesity or MetS are noted to have low HDL-C regardless of age in some populations, probably based on genetic susceptibility. Gender also has an impact on HDL-C level, lower in females than males in general. Then, triglycerides and total cholesterol levels increase with age.

In addition to age and gender genetic factors, obesity and ethnicity play a role in developing metabolic syndrome, with some ethnic groups being significantly at less risk for developing MetS, most probably due to genetics and differing life styles. Therefore, prevalence of metabolic syndrome should be adjusted for age, gender and ethnicity. Differing cluster patterns may have different impact on outcomes of MetS and hence different treatments might be required.

Although it is not clear whether insulin resistance is the cause or the effect of MetS, there is association with insulin resistance. Most of all the components of clustering factors of MetS is seen in subjects of high waist circumference with ethnic specificity. MetS association with central adiposity, high BMI and ethic specificity and increasing trend with aging seems to indicate a disease of adipose tissue failure. Subcutaneous tissue might help to store fat to some extent but due to time or genetic factors, hyperglycemia, high BMI, hormonal factors and gender may exceed storage capacity thus transfer fat to abdominal subcutaneous tissue and later visceral organ capacity. These are sources of fatty acid fueled by the liver when insulin resistance is present. This hypothesis is useful in the implementation of strategies to prevent type $2 \mathrm{DM}$ and coronary artery and cardiovascular disease. The implementation of intervention therapies such as health education has not reached to grass root however especially in developing countries fighting not only infectious but also non-infectious diseases of urbanization. The health sector would require use of social media, national and political and economic sectors involvement are mandatory to educate the public on healthy food and change of life style.

The purpose of attempts of description and definition of MetS are to prevent the development of type $2 \mathrm{DM}$ and coronary artery diseases.

Among the various definitions of MetS, NCEP-ATP111 is most significantly associated with coronary artery disease than other definitions. However, all the definitions are good predictors of type 2 DM because all are significantly associated with type 2 DM. Presence of MetS in obese subjects and diabetics are significantly associated with coronary artery disease related mortality and all-cause mortality whereas those without MetS are not. The presence of MetS in type 2 $\mathrm{DM}$ is a determining factor for development of both macro-vascular and microvascular diseases. MetS without type $2 \mathrm{DM}$ also has increased propensity for developing stroke. Although individual components of MetS such as hypertension, dyslipidemia and DM are well accepted traditional risk factors for developing coronary artery disease MetS by clustering those risk factors has significant additive effect and as high cut-off points of individual component of MetS have higher risk of developing cardiovascular diseases help enhancing preventive measures. Therefore screening or efforts to define MetS is to identify the individual at high risks for developing DM and coronary artery disease. Although obligatory factors are not encouraged to diagnose metabolic disease, high waist circumference is a sensitive tool to probe the metabolic syndrome and practice of measuring waist circumference should be encouraged individually and in the health clinics. As individuals with MetS have risk of developing type $2 \mathrm{DM}$ in 5 to 10 years, it is necessary to identify which factors contribute to this increased risk. As combined high triglycerides and low HDL-C is a hall mark of diabetes, vigorous intervention measures can be carried out for those individuals in whom MetS is defined by high triglycerides and low HDL-C.

More efforts are necessary for further research of etiology, pathophysiology and pathogenesis of MetS. Further studies on mechanisms of development of central obesity are mandatory. Factors which determine the development of subcutaneous fat and visceral fat should be further examined. Extended studies on factors which control insulin signals in muscle and liver cells might be helpful to reduce or retard or prevent development of insulin resistance. 
BMI and waist circumference have very good correlation with Mets. Obesity, one of the risk factors for development of Mets is increasing globally. It is necessary to discern common global factors or developing obesity as globalization and urbanization are apparently affecting metabolism of individuals. The global rise in prevalence of type $2 \mathrm{DM}$ is beyond genetic factors. 